\title{
New Load Balanced Multi-Path Dynamic Source Routing Protocol for Mobile Ad-HOC Network
}

\author{
Lokesh Malviya \\ M.Tech Scholar \\ BIST Bhopal \\ India
}

\author{
Akhilesh A. Waoo \\ Associate Professor \\ BIST Bhopal \\ India
}

\author{
Sanjay Sharma, PhD. \\ Professor, MANIT \\ Bhopal (MP), \\ India
}

\begin{abstract}
Mobile ad hoc networks consist of mobile wireless devices which autonomously organize their infrastructure. Its performance depends on the value of different parameters, like number of nodes and mobile connections. Multi-path routing allows building and use of multiple paths for routing between a source-destination pair. It exploits the resource redundancy and diversity in the underlying network to provide benefits such as fault tolerance, load balancing, bandwidth aggregation, and improvement in QoS metrics such as delay. In this proposed work is converting Dynamic Source Routing Single path Protocol into Multi-path Routing with concept of Load balancing. The source node maintains up to 5 paths in its ROUTE CAHCE which it receives as a result of Route Discovery process and Target node will receive RREQ packets and send RREP packets for first 5 requests received. Currently it is replying only first RREQ packet received and rejects the remaining. Hence its can improve the DSR performance.
\end{abstract}

\section{Keywords}

Ad-hoc networks, wireless networks, mobile networks, routing protocols, simulation, performance evaluation.

\section{INTRODUCTION}

Mobile Ad Hoc Network has emerged as a major area of research for both academic and industrial sectors. Simply stating, a mobile Ad HOC Network (MANET) is one that comes together as needed, not necessarily with any support from the existing infrastructure or any other kind of fixed stations. As for the mobile operation, ad hoc network are basically peer-to-peer multi-hop mobile wireless networks, where information packets are transmitted in a store-andforward manner from a source to an arbitrary destination, via intermediate nodes.

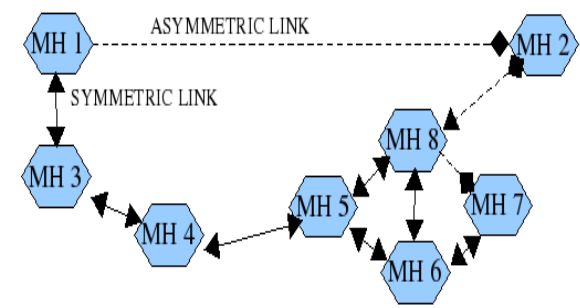

Figure 1 Ad Hoc Network
In figure 1 as the Mobile Hosts $(\mathrm{MH})$ move, the resulting change in network topology must be made known to other nodes so that outdated topology information can be updated or removed. For example, as MH2 in fig. 1 changes its point of attachment from MH3 to MH4 other node's part of the network should follow this new route to forward packets to $\mathrm{MH} 2$.

The issue of symmetric and asymmetric links is one among the several challenges encountered in a MANET. Another important issue is that different nodes often have different mobility patterns. Some MHs are highly mobile, while others are primarily stationary. It is difficult to predict a MH's movement and pattern of movement.

Multi-path protocols have a relatively greater ability to reduce the route discovery frequency than single path protocols, among the on-demand protocols. In the MANET On-demand multi-path protocols discover multiple paths between the source and the destination in a single route discovery. So, a new route discovery is needed only when all these paths fail.

\section{DSR PROTOCOL EXPLANATION}

Dynamic Source Routing' (DSR) is a routing protocol for AdHOC networks. It is similar to AODV in that it forms a route on-demand when a transmitting computer requests one. However, it uses source routing instead of relying on the routing table at each intermediate device. This protocol is truly based on source routing whereby all the routing information is maintained (continually updated) at mobile nodes. The DSR protocol is composed of two mechanisms that work together to allow Route Discovery and Route Maintenance of source routes in the ad hoc network:

\subsection{Route Discovery in DSR}

When node $\mathrm{S}$ wants to send a packet to node $\mathrm{D}$, but does not know a route to $\mathrm{D}$, node $\mathrm{S}$ initiates a route discovery. Source node $\mathrm{S}$ floods the network with route request (RREQ) packets (also called query packets).Each node appends its own address in the packet header when forwarding RREQ. Destination D on receiving the first RREQ sends a Route Reply (RREP). RREP is sent on a route obtained by reversing the route appended to receive RREQ.RREP includes the reverse route from $S$ to $D$ on which RREQ was received by node $\mathrm{D}$. 


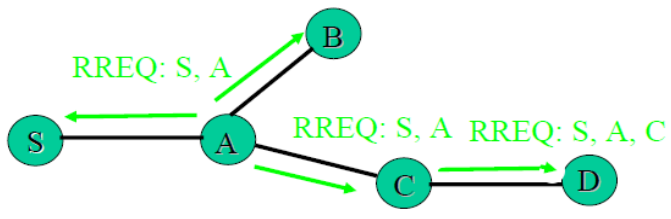

Figure 2.1 Route Request

\subsection{Route Maintenance in DSR}

The path maintenance method assures that the routes saved in the path cache are validated. We assume that all nodes wishing to communicate with other nodes within the ad hoc network are willing to participate fully in the protocols of the network. Each node participating in the network should also be willing to forward packets for other nodes in the network. if the network topology has changed such that it can no longer use its route to D because a link along the route no longer works. When Route Maintenance indicates a source route is broken, $\mathrm{S}$ can attempt to use any other route it happens to know to D, or it can invoke Route Discovery again to find a new route for subsequent packets to D. Route Maintenance for this route is used only when $S$ is actually sending packets to D.

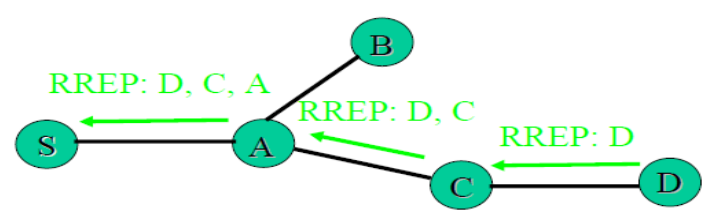

Figure 2.2 Route Reply

\section{MULTI PATH DSR (MP-DSR)}

For this study, we selected five multi-path DSR variants with different path selection schemes. MP-DSR or Multi-Path Dynamic Source Routing introduced in [1], tries to find multiple disjoint paths from given source to a destination while guaranteeing that these paths altogether can satisfy a given end-to-end reliability. The destination replies with a RREP for the first received RREQ (primary route over shortest path) and all subsequently received RREQs over linkdisjoint paths. The source node uses the primary path and stores all alternatives in case of a route error. The shortest backup route always replaces a currently broken route. MDSR does not modify the forwarding of the RREQs by intermediate nodes as it is specified in [2]

\section{RELATED WORKS}

Christos Tachtatzis and David Harle propose The one camp supports the belief that multi-path routing is indeed advantageous compared to single path counterparts and the other contends that the improvements not significant, if at all. For the performance evaluation of the two counterparts, Dynamic Source Routing (DSR) protocol is compared against a modified version of DSR which exploits multiple node disjoint routes. The comparison of the two routing schemes is carried out using the Network Simulator ns-2, and the comparison is performed for static and mobile scenarios. The performance is quantified and it is shown that the proposed routing scheme outperforms the uni-path counterpart.[3]

V.Ramesh, Dr.P.Subbaiah, N.Sandeep Chaitanya, K. Sangeetha Supriya Proposed Multi-path routing protocol is one of the approaches used to have less overhead, better bandwidth cost by distributing the load among a set of paths However, due to interfering in the channels of the paths, multi-path increases the end to end delay and do not work well in highly congested networks. A congestion aware multipath dynamic source routing protocol. We generate a set of disjoint multi-paths and handle the problem of end to end delay using the correlation factor measurement. A set of path selection criteria have been used to improve the end to end delay [4]

Shun Liu Jian Liu propose a delay-aware multi-path source routing (DMSR) protocol to provide QoS support for real time multimedia applications in wireless ad hoc networks. Based on local information, node delay is calculated as metric for route path selection in the DMSR protocol. The metric takes into account the number of the neighbor nodes of the forwarding nodes, the channel busy time and the number of packets in the send buffer. Simulation analyses and results show that the DMSR protocol can reduce the average end-toend delay and meet the demands of real time multimedia services compared to the dynamic source routing (DSR) protocol and the improved DSR.[5]

Anil Rawat, P. D. Vyavahare, A. K. Ramani propose Ondemand routing protocols for Mobile Ad-hoc Networks (MANET) include Dynamic Source Routing (DSR), which is capable of discovering multiple routes. Secured Routing Protocol (SRP) [2] on the other hand provides mechanism for secured route discovery, even under adversarial conditions. The paper proposes integration of SRP features in DSR to gain security features for MANET routing requirements for the route discovery phase. Concurrent use of multiple paths for data transmission phase and its implications on performance is also evaluated [6]

\section{PROPOSED WORK FOR NEW MP- DSR}

In this proposed Work, We modified the route cache with a new attribute RouteID and Introduced a Load Table. Load Table has two attribute RouteID and Packet count. The source node maintains up to 5 paths in its ROUTE CAHCE it receives as a result of Route Discovery process and Target node will receive RREQ packets and send RREP packets for first 5 requests received. Currently it is replying to only first RREQ packet received and rejects the remaining. 
Route Discovery this process is initiated when there is no path to given target node and the total no. of paths for a target node are less than 5 at any point in time during transmission. In both the above situations, source node prepares a RREQ packet and broadcast it to the neighbors. The neighbors will do the same after making an entry into ROUTE REQUEST TABLE. These multiple copies of RREQ packets move through the network through all possible paths to target node. Finally, the - target node replies for the first five RREQ packets and in this manner source node receives at most 5 different routes to the target node. It may be possible that less than 5 RREQ packets reaches target node as due to mobility of nodes topology of network continuously changes. But by enabling target node to reply more than one RREQ and at most 5 RREQ's, the multipath feature is incorporated. We did not change in original route maintenance mechanisms.

\subsection{Load Balancing}

For ongoing transmission, packet will be forwarded on round robin manner amongst the 5 paths (or available multiple paths less than 5).

When originating a new packet, a node must perform the following steps:

(i) Search the Route Cache for a route to the address given in the destination address field in the packet's header.

(ii) If no such route is found in the Route Cache, then perform Route Discovery for the destination address.

(iii) If total routes available in Route Cache for given destination address are more than 5 , select the 5 shortest routes.

(iv) Identify path to transmit packet on, considering load balance.

(v) Transmit the packet to the first-hop node address given in the identified source route.

The route maintenance activity will also be affected as now more than one path breaks can occur due to mobility. This will generate great result as compare to DSR and MP-DSR

\section{SIMULATION MODEL}

NS2 simulator is adopted in this paper to assess the performance of the proposed modified concept and compare with the traditional DSR based mobile ad-hoc network. We also evaluated the protocol using the following matrices:

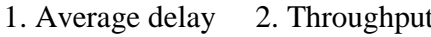

\section{CONCLUSION}

Analyze of DSR routing protocol is the biggest problem in ad hoc networks is due to mobility, as link break occurs very frequently. In the proposed work, a modification will be introduced in the existing DSR protocol by using multiple paths instead of a single path as used in the original DSR. By using multiple paths for transmission, this problem can be reduced which is used for path selections. Applications can specify their end-to-end reliability requirements to control the routing failure probability. With our algorithm End-to-end reliability is also maintained throughout the whole transmission life time. Simulation results show that our modified DSR can offer higher and more consistent success delivery ratio than DSR.

\section{REFERENCES}

[1] R. Leung, J. Liu, E. Poon, A.-L. C. Chan, and B. Li. "MPDSR: A QoS-Aware multi-path dynamic source routing protocol for wireless ad-hoc networks" In LCN, pages 132-141. IEEE Computer Society, 2001.

[2] [2] Bastian Blywis ,Mesut Günes ,David J. H. Gutzmann ,Felix Juraschek " A Testbed-based Study of Uni- and Multi-Path Dynamic Source Routing in a WMN", IEEE 978-1-4244-9229-9/10,2010

[3] Christos Tachtatzis and David Harle "Performance Evaluation of Multi-path and Single-path Routing Protocols for Mobile Ad-Hoc Networks" IEEE SPECTS 2008

[4] V.Ramesh,Dr.P.Subbaiah ,N.Sandeep Chaitanya , K. Sangeetha "Performance Comparison of Congestion Aware Multi-Path Routing (with Load Balancing) and Ordinary DSR", IEEE 978-1-4244-7932-0/10 2010

[5] Shun Liu Jian Liu Delay-aware, "Multipath Source Routing Protocol to Providing QoS Support for Wireless Ad Hoc Networks" Communication Technology (ICCT), 2010 12th IEEE International Conference on,Pages: 1340 - 1343.

[6] Anil Rawat, P. D. Vyavahare, A. K. Ramani "Enhanced DSR with secured multi-path route discovery and concurrent data transmission",Proceedings - 2006 14th International Conference on Advanced Computing and Communications, ADCOM 200,pages:612-613.

[7] Ali F. Almutairi, Senior Member IEEE, and Taher M. ElHendawy "Performance Investigation Using Different Software of Dynamic Source Routing in Ad-Hoc Networks",GCC Conference and Exhibition (GCC), Page(s): 307 - 3102011 IEEE

[8] Sangeeta Biswal, "Study of DSR Routing Protocol in Mobile Ad-hoc Network" 2011 International Conference on Information and Network Technology IACSIT Press, Singapore IPCSIT vol.4 (2011)

[9] Geetha jayakumar and G Gopinath "Performance comparison of Two On- demand routing protocols for Ad-hoc Networks based on random way point mobility model" Department of Computer Science, Bharathidasan University, Triuchirapalli- Pages :620 023.

[10] Stefano Basagni, Marco Conti, Silvia Giordano, Ivan Stojmenovic "Routing Approaches in Mobile AD HOC Networks"JAN 2005 ,DOI:10.1002/0471656895.ch10

[11] Sarala.P , Kalaiselvi "Multipath Dynamic Source Routing with Cost and Ant Colony Optimization for MANETS ",International Journal Of Applied Engineering Research, Dindigul Volume 1, No1, 2010.

[12] D. B. Johnson and D. A. Maltz, "Dynamic source routing in ad hoc wireless networks," in Mobile Computing, T. Imielinski and H. Korth, Eds. Kluwer, 1996, vol. 353.

[13] Lee. S and Gerla.M "Split Multipath Routing with Maximally Disjoint paths in Ad Hoc Networks". In Technical Report in University of California, 2000

[14] Rashida Hashim, Qassim Nasir, Saad Harous, "Congestion Aware Multi-path Dynamic Source Routing Protocol (CAWMP-DSR) for Mobile Ad-Hoc Network" in Proceedings of MoMM2007, pp. 199-205. 